\title{
The cost of visit-based home care for up to two weeks in the last three months of life: A pilot study of community care based at a hospice-at- home service in South East of England
}

Purpose: The cost of visit-based community care based around a 24/7 hospice-athome (HatH) service in the last 3 months of life was assessed.

Methods: Thirty families completed a health and social care diary of at-home visits over two-weeks following contact with the HatH night service.

Findings: Diaries captured 333 days of care provision, averaging 11 diary days per family, 708 health care professional and carer visits, lasting 604 hours at a cost of $£ 20,192(\$ 24,946)$.

Conclusions: HatH care, integrated with community support, seems an economic proposition but highlights the complexities of assessing cost of end of life care.

Keywords: Hospice-at-home, costs, Community, End-of-life

Word count: 4546 


\section{Introduction}

Extensive evidence shows that over $50 \%$ of people say they prefer to die at home (Gomes, Calanzani, Curiale, McCrone, \& Higginson, 2013; Gomes, Calanzani, Gysels, Hall, \& Higginson, 2013), especially if they can be assured that symptoms are well managed and family burden reduced. However, in England, only $23.5 \%$ achieved this in 2016 (National End of Life Care Intelligene Network, 2018; Public Health England [PHE], 2018), with up to 50\% of individuals dying in hospital (Marie Curie Cancer Care, 2014; PHE, 2018). While interpreting survey data on preferred place of death can be difficult often due to poor survey completion rates and changes in circumstances (Hoare, Morrise, Kelly, Kuhn, \& Barclay, 2015), there remains a discrepancy between preference and actual place of death, suggesting more could be done to meet patient's wishes to die in their preferred location (Bekelman et al., 2016). This is despite the Department of Health's [DoH] end of life (EoL) guidance (DoH, 2008), which stresses individual choice in where to die. The DoH encourages the UK National Health Service (NHS) to provide 24-hour at-home care to improve quality of life and death and to enable more people to die at home (DoH, 2008).

Dying at home can be difficult to achieve for several reasons, such as the lack of a spouse or family support (around 50\% of people at EoL die without a partner), and dispersed families may no longer be able to offer adequate support (Gomes \& Higginson, 2006). Moreover, people are living longer and increasingly experiencing problems associated with frailty and dementia, which can precipitate transfer to care homes and increase the likelihood of admission to hospital when acute problems develop (Bentur, Renizky, Balicer, \& Eilat-Tsanani, 2014). Given such demographic and age-related changes, establishing the cost of dying at home is important for 
resource planning and health budgeting as well as for developing new models which balance care in appropriate settings with economic necessity.

The importance of providing optimal palliative care in the home that supports people with advanced illnesses and their families who wish to stay at home, is particularly topical for an ageing population. Excellent home care has been shown to significantly improve the symptom burden on patients without increasing caregiver grief (Gomes et al., 2013). The cost of EoL care varies across the UK, and internationally, with hospital care being the most expensive and home care more cost-effective for most health systems (Bentur et al., 2014; Georghiou, Davies, Davies, \& Bardsley 2012; Marie Curie Cancer Care, 2015; Woodman, Baillie, \& Sivell, 2016). However, a Cochrane Review, which incuded six studies assessing cost-effectiveness, was inconclusive (Gomes et al., 2013), and the majority of these studies were from other countries and small. Palliative and EoL cost effectiveness studies have been hampered by a number of methodological limitations including insufficient accounting for informal caregiver costs, lack of attention to quality of care outcomes, a narrow focus on included costs, different unit cost calculations, short timeframes and the heterogeneity of interventions (Butler et al., 2018; May \& Cassel, 2018). Finally, the sensitivity of conducting research in this area has meant that many studies have concentrated on retrospective records, especially regarding hospital care, single index hospital admissions and decedent cohort studies (May \& Cassel, 2018).

Given such limitations, studies that provide real time UK information gained from patients and/or family carers can make an important contribution to the literature. Clarity about the unit cost calculations utilized alongside the scope and content of the 
intervention being evaluated is critical to better understanding, especially since there are important differences between models of community based/hospice-at-home care which are currently being assessed to identify optimal methods of provision (Butler et al., 2018).

The data from this study derive from an evaluation of the quality and effectiveness of a Hospice-at-Home (HatH) night service (Sixsmith, Ward, \& Youell, 2017). The service is a charitable organization providing 24-hour care for parts of the Thames Valley and East of England, UK. In 2017, clinical staff visited and cared for 1,760 patients and their families at home. Care provision included medical, technological and nursing care, and provided for the emotional, practical and informational needs of patients and family members, with $85 \%$ of clients who wished to die at home achieving their wish.

\section{Methods}

The overall study was a mixed methods evaluation (Sixsmith et al., 2017). One aim of the evaluation was to establish the day-to-day costs of HatH care of patients judged to be within three months of EoL, and to reference these costs to in-hospital care costs. To address this issue, an in-house tool, in the form of a night service template (see Figure 1), was used to capture contacts made to the service overnight. Subsequently, the night and day care costs were established in a bespoke health care diary for some of those contacts.

Insert Figure 1 
Ethical approval was gained from the School of Health Research Ethics Committee, at the university leading the evaluation (RGHaHC-04.05.2016), and governance was gained from the hospice's clinical governance committee, as was appropriate for the evaluative nature of the project. All participants were informed about the study and written consent was obtained for the diary data collection and access to routinely collected data.

\section{Night service template}

A night service template was designed with HatH staff to collect data from all telephone calls made to the HatH service, overnight, between 21.15 and 7.15, for 145 nights between July-December 2016, to ascertain: reason for a call; necessity of a night visit; or whether telephone advice provided adequate support without requiring a visit. If a visit occurred, the team assessed, to the best of their ability and professional judegement, whether the patient was probably within the last three months of life, and then whether the patient and/or family were willing to collect diary data for up to two-weeks following the visit.

\section{Health care diary}

The two-week diary was co-designed with HatH staff to record the range and frequency of services visiting patients/families at home following any HatH visit, day and night (or until death occurred). Using the diary patients/families recorded all health care visits made daily by: General Practitioner; District Nurse; Specialist Nurse; Hospice Nurse; Marie Curie/ MacMillan; Social Services/Formal Carers; and an open text option for 'Others', which included visits from chiropodists, occupational therapist, and physiotherapists, to capture all other professionals. The 
diary was pre-tested with staff and families $(\mathrm{N}=10)$ to ensure ease of understanding. Following this, the diary was simplified, and the revised version was issued to a further eight participants and completed by four. Further minor changes were made to the format and wording of the instructions.

In agreement with the HatH staff, the diary was not given to patients or family members considered to be especially vulnerable or going through a particularly difficult stage in the illness trajectory. This was a subjective decision based on prior knowledge of the family and a judgement as to whether asking to keep a diary was appropirate or if families may be experiencing extreme difficulties in coping, this was assessed by experienced and specialist nursing staff. All patients/families visited by the night staff were considered for the diary and those who were offered and accepted were supported through a phone call each week by HatH staff to encourage completion of the diaries.

Diaries were left with the patient/family to complete and were collected by the HatH staff or returned via freepost envelope. On data transfer, patient ID numbers were used to preserve anonymity. Anonymised records were cross referenced with HatH records to capture age, gender and date of death information, where appropriate.

Between July-December 2016 the diary was distributed to 35 accepting family carers/patients, 17 diaries were returned (48\% response rate). The diary data provided: valuable information on all the range of services working with patients both during the day and night-time; time spent in the home by services; and the cost of service provision was then calculated. To develop a stronger data set, a second phase of data 
collection was undertaken using the same selection criteria and procedure. Between June-August 2017, 27 diaries were distributed, and 13 returned (48\%), making 30 diaries available for analysis. The demographic profile of the two cohorts was similar, $53 \%$ were male patients in the original cohort compared to $54 \%$ in the second cohort. The mean age of patients was 78 and 74 respectively, and $53 \%(n=9)$ of patients died during the initial data collection period and $38 \%(n=5)$ in the second phase. Of the 30 patients, 14 died during the data collection period. Data was entered into Excel and IBM SPSS v26 (IBM, 2018) for descriptive and statistical analysis (Indepented Samples t Test using Levene's Test for Equality of Variances).

A cost tariff analysis was undertaken to estimate the costs of visits from health and social care professionals. Average costs were predominantly identified through the Personal Social Services Research Unit (PSSRU) costs for health and social care 2015/16 (Curtis \& Burns, 2015). Costs not available through the PSSRU were identified through service specific websites (Data Gov UK, 2015; MacMillan Cancer Support, 2016; Payscale Care Worker Salary, 2017; Payscale podiatrist salary, 2017; UK Care Guide, 2016). Where more than one cost was provided per hourly rate (e.g. due to different band grades or salary ranges) an average of the costs was calculated.

Rates for the HatH staff were provided by their finance team. These included 'oncosts', in-line with PSSRU cost calculations. An average cost for HatH staff across bands 3-7 and the day and night teams was calculated; this average was used to provide a 'best fit' with the PSSRU costs, and included calculations of the hourly cost for each Band, plus additional costs (National Insurance, pension contributions, and $20 \%$ salary add-on cost). The constitution of the night team was one band 7 clinical 
nurse specialist (0.64wte), three band 6 nurses (1.76wte) and three band 3 health care assistants (1.38wte). Two staff were on each night. Of the five day-teams covering the area, the mix of staff was similar.

\section{Results}

Summary data from the 30 diaries is presented in Table 1 . This shows the total number of visits made by each service category, the proportion each category contributed to overall care, and total time each professional spent with a client and the estimated cost of this care.

Insert Table 1

Overall, 333 days were recorded by the diaries, averaging 11 days per diary (range 120, while participants were asked to record all visits for 14 days, three provided data for a longer period of 16, 18 and 20 days). There were 708 visits lasting a total of 604 hours and costing $£ 20,192$ ( $\$ 24,946)$. This can be calculated as average costs of $£ 28.5$ (\$35.2) per visit, $£ 60.6(\$ 74.8)$ per day, and $£ 673.1$ (\$831.6) per person over an average of 11 days. The lowest cost of care for an individual reported by the diaries was $£ 33.2$ (\$41.0) for one visit prior to a patient’s death, and the highest care for an individual was $£ 2,276.3$ (\$2,812.3) over 20 days and 162 visits (made by district nurses and HatH staff, and formal carers, who visited several times each day). In order to understand whether this cost was high due to the greater number of diary days recorded, it was also reviewed in terms of the average cost of care per day. An average daily cost was calculated at $£ 113.8$ (\$140.6) for the 20 days, which was the second highest daily average care cost. The highest average daily cost of care was 
$£ 127.6$ (\$157.6), this was for a patient who recorded 11 diary days, costing a total of $£ 1,403.4$ (\$1,733.8), with over two-thirds of this individual's care provided by overnight specialist cancer nurse care and care from the HatH staff (see Table 2 for full cost of care, number of diary days and average per day, per patient). The highest daily cost was $£ 578.1$ (\$714.2) for four visits; especially high due to a visit of two hours made by a General Practitioner. The lowest daily cost was estimated as under $£ 5$ (\$6.2), for 30 minutes of formal care provision - this indivudiaul received care from other services on other days, but this represented the lowest amount of care provided in a single day. The highest daily cost does not include one patient's reported hospital admission, the costs for which were not part of the home care received, although this would have contributed to the total cost of care. The number of visits made per day and long visits by high cost services are high cost outliers in providing EoL care at home.

Insert Table 2

The cost of care for the 14 patients who died during the data collection period (thereby representing some of the most complex cases) was $£ 11,709.1(\$ 14,466.2)$, averaging $£ 836.3(\$ 1,033.2)$ per individual. This compares to $£ 8,483.2(\$ 10,480.7)$ for the cost of care for those who did not die during the data collection period, averaging $£ 530.2$ (\$655.1) per individual $(\mathrm{n}=16)$. An Independent Samples t Test was conducted using IBM SPSS v26 (IBM, 2018) to test the null hypothesis that mean daily care costs were equal between those who had died during the data collection period and those who had not. A significant difference was identified $(\mathrm{t}=2.919, \mathrm{df}=28$, $\mathrm{p}=0.007)$. The results indicate that those who died had a higher average daily cost of 
care (mean average daily care cost for those who died was: $£ 78.95$ (\$97.54), 95\% CI, range $£ 64.11-£ 93.78$ (\$79.21-\$115.86); and for those who did not was: $£ 46.20$ (\$57.08), 95\% CI, range $£ 27.88-£ 64.53$ (\$34.44-\$79.72) - see Figure 2).

Insert Figure 2

Our estimated costs do not include the travelling time for health and social care professionals as it was not possible for families to determine this. It did not appear feasible to ask families to report on travel times and destinations of visitors, as this may have been difficult to collect, and could have reduced participation in the project. In a pilot of the diary, families reported that too many options would be challenging to complete and the diary was designed to be quick and easy to use. We therefore used the night service template criteria to determine the average journey time which was determined from the travel time recorded per night and averaged across the number of visits made. This was 1 hour 14 minutes per patient visit, with an average distance of 24.4 miles per visit and an average cost of $£ 10.99$ (at $£ 0.45 \mathrm{p} / \mathrm{m})(\$ 13.58$ at $\$ 0.56 \mathrm{p} / \mathrm{m}$ ) plus an average salary cost for travel time of $£ 54.63(\$ 67.49)$ per journey. At a average of 2.1 visits per 24 hours reported by the diaries from all Healthcare Professionals (including hospice staff), assuming a travel time and cost similar to that of the hospice night staff, this would add $£ 114.72(\$ 141.73)$ to the daily cost of care.

Formal carers/social service staff provided the highest number of contacts at $47 \%$ of all visits made during the study period, hospice staff provided $25 \%$ and District Nurses $17 \%$. Together, these services provided $89 \%$ of all the visits recorded by the 
diaries. While formal carers/social services accounted for $47 \%$ of all visits, this constituted only $11 \%$ of the total cost of care and a total of 231 hours of visit time. HatH day and night staff made 175 visits/contacts (25\% of total contacts) and accounted for $37 \%$ of the total cost of care - the highest cost across all the services and 165 hours of visit time. The District Nurses (daytime only) carried out 117 visits ( $18 \%$ of all contacts) and accounted for $18 \%$ of the total cost of care, and 64 hours of visit time.

Table 3 (below) reports on the number of recorded visits made to patients by Healthcare Professionals for both those who died during the data collection period and those who did not. In line with the increase in costs for people in the last days of life, the number of visits made by these services is also shown to be higher. On average, those in the last days of life had 1.5 visits from a General Practitioner and 4.3 visits from a District Nurse, compared to those not in the last few days of life which were 0.9 and 3.6 visits respectively. This also compared with the HatH service which attended individuals on average on 7.4 occasions when in the last few days of life compared to 4.4 visits when not so near to death. Total visits made by all health and social care professionals also showed an increase, with an average per individual of 33.1 for those in the last few days of life compared to 15.3 for the other patients.

Insert Table 3

The cost of care for general practitioner visits was relatively high in comparison to numbers of visits made, accounting for $14 \%$ of the overall costs but only $5 \%$ of visits, and 14 hours of visit time. The overnight care provision by MacMillan/Marie Curie 
nurses accounted for $14 \%$ of total costs but only $2 \%$ of visits, and 99 hours of visit time (relatively high as they were overnight visits).

In comparison to at-home care costs, according to the PSSRU 2015/16, the cost of an inpatient specialist palliative care bed lies between $£ 289-£ 540$ (\$357-\$667), with a national average of $£ 397$ (\$490) a day (Curtis \& Buris, 2016). Once admitted, the length of stay will also vary depending on the care and treatment required. There will also be an additional cost for an ambulance call out and admission, for example in an accident and emergency department, which would add on average a further $£ 231$ (\$285) to the costings, resulting in an average of $£ 628$ (\$775) for a day long hospital stay.

\section{Discussion}

This study aimed to address some of the challenges of collecting cost data for EoL care, and while presenting findings from a small sample, it used a diary method to collect up-to-date data of Healthcare Professional visits, adding a potential approach to counter criticisms of retrospective methods (Gomes et al., 2013). This may not be a method without issues, as it relies on the carers to record the visit, and as was noted, sometimes the length of time of a visit was not recorded. However, it does present a reasonably comprehensive picture of the range of services who make at-home visits and the number of visits which occur. The picture presented through the diary data is one of complex needs being met by a number of different health and social care providers, most notably formal carers, the HatH staff and district nurses. As highlighted by a Cochrane review (Gomes et al., 2013) there are very few prospective studies of EoL costs for at-home care in the literature, and of these a minority are from the UK, all 
with small numbers of subjects (Publich Health Englnad, 2018). Furthermore, studies often rely on retrospective approaches potentially suffer from memory inaccuracies or were reliant only on hospital or General Practitioner records (Gomes et al., 2013).

Further issues which make the assessment of cost challenging across studies, is highlighted by a review of estimated costs of palliative care in the UK, which identified the considerable spread of costs across different models of care available (Gardiner, Ryan, \& Gott, 2018). While the diary approach provided a comprehensive picture of the range of services visiting, it was not possible for families and patients to record the band of the staff member attending, or the travel time/cost associate with their visit. The costs of medication and/or equipment were also not included. However, this provides an area where future research could build upon the diary method, adding in cross checks with service providers or requests for staff to complete visit logs.

This study attempts an assessment of the cost of visit-based at-home care in patients judged to be in the last three months of life. In line with previous research, it suggests that EoL care at home can be cost effective when compared to hospital care (Bentur et al., 2014; Lustbader et al., 2017). The costs quoted were based on salary costs per hour and Healthcare Professional/carer travel costs were not included as it was not possible for families to record this. However, our estimate of $£ 114.7(\$ 141.7)$ a day based on the night team travel costs is likely to be an overestimate, as some Healthcare Professionals and carers were on a lower salary scale and also day staff would plan their 'round', minimizing distances to travel, and be more cost effective . The salary costs of an average of $£ 60.6(\$ 74.8)$ per day plus travel costs still makes home care an economic alternative. However, estimating the cost of health and social 
care, and particularly hospital care is complex (Gardiner, Ryan, \& Gott, 2018; PHE, 2017). The PSSRU provides the most up-to-date data available and is a widely used and valued resource (Curtis \& Burns, 2016). For this reason, the PSSRU was used to provide the costings for hospital admissions and hourly rates for health care services, excluding medications and equipment.

Costs of care were higher for those who were at EoL, with a mean average daily care cost of $£ 78.95$ (\$97.54), with a statistical difference found between the mean average daily costs of those who had died and those who had not. This was associated with greater health needs, averaging 7.4 HatH nurse visits compared to 4.4 for those who remained alive during the data collection period. Overall, the average total visits per individual who died was 33.1 visits compared to 15.3 for the other patients. This supports findings of a Nuffield Trust study of cancer patients (Georghiou \& Bardsley, 2014), which showed costs could increase towards the EoL, and that visits from General Practitioner and district nurses increased both in frequency and time spent with the patient. The findings from this current study, therefore, add to the evidence base that EoL care can be more time and cost intensive the closer a person is to death. Evidence also suggests that at-home care may reduce hospital admissions, saving money and unnecessary upheaval for families (Robinson et al., 2015). A large retrospective literature search of data examining palliative care services for cancer and comparing home to hospital care and costs concluded that, in general, home care was under utilised but cheaper than hospital care, and that prospective patient-centred data is crucial if one is to move toward optimising EoL care globally (Langton et al., 2014). A Public Health England (2017) report on cost-effective commissioning at EoL, identified considerable differences in the way research calculates costs, and the resulting challenge of 
comparing these costs across studies and different interventions (Georghiou \& Bardsely, 2014).

\section{Limitations}

Our study has several caveats. This study tried to put in place measures to ensure the accuracy of the diary day (support and checks offered by the HatH staff), however, this was largely self-reported, and it is not possible to ascertain whether all visits were recorded and their lengths accurate. There was a balance to consider in ensuring that patients and families were not put under undue pressure, particularly at a time when many were experiencing considerable distress.

The assessment of costs is complicated by the complexities of EoL care, with the mix of patients and conditions strongly influencing the resultant associated costs (McCaffrey et al., 2013). This is evidenced in the current findings through the range of visits made to patients which varied both in terms of number of visits but also by service. The data also suggests that for an individual patient, the cost of care by Healthcare Professionals in the home is surprisingly inexpensive when compared to hospital inpatient stays. Although it should be noted that on some days no visits were recorded, while on others, multiple visits by multiple professionals were made, for example HatH staff could make two visits alongside a district nurse and formal carer. While the average daily cost is relatively low, this fluctuated according to individual needs, reflective of other studies (McCaffrey et al., 2013).

The exclusion of vulnerable patients from the study may have had an impact on the overall reported costs, with more complex needs cases possibly having been excluded, 
leading to an underestimation of $\mathrm{HaH}$ costs. Furthermore, the collected data does not take account of the number of other patients seen by HatH staff and other Healthcare Professionals during the days monitored, and cost of care per person visited will depend on the number of families visited relative to the salary costs which are a fixed cost, irrespective of the number of patients seen on a shift.

Some accountable costs were not considered, primarily the cost of informal care provided by family and friends, equipment and medications. This has been addressed in a large retrospective study (Rowland, Hanratty, Pilling, van den Berg, \& Grande, 2017), using Office of National Statistics data pertaining to relatives of patients dying of cancer. This suggested nearly 10 hours of care was provided per day by relatives and friends, plus 'out of pocket' expenses of $£ 370$ (\$457) over the three months (Rowland et al., 2017). The main type of care was social/emotional support, although many contributed to the practical side of care. A Marie Curie report (2015) estimated that informal carers save the UK economy $£ 132$ (\$163) billion every year and for those specifically at EoL with a form of cancer, informal care saves the economy $£ 219$ (\$270.5) million annually. However, there is also a 'hidden' cost for carers’ health and wellbeing, with many experiencing a negative impact on their emotional resilience, something future studies could explore further in relation to the cost of care at EoL.

A further complexity which makes it difficult to extrapolate the costs from this study to other HatH organisations, is that their staff mix and costs are likely to be different. Nevertheless, we provide evidence that caring for patients at home may costs less than inpatient hospital care and can be used as part of the information needed to 
assess requirements for best home palliative care. We also highlight the complexity of conducting cost comparisons with other community models and hospital care and emphasizes the need for greater standardization of research in this area. The review on cost-effective commissioning by PHE (2017) supports this by identifying the need for comparative control groups, adequate sample sizes, and considerations of quality of care alongside cost of care, all of which are challenging to address with patients at EoL, but which future studies could consider addressing.

\section{Conclusion}

The diaries provided an important insight into the range of services engaging in patient care at EoL, which is presented as a complex interaction of multiple services with formal carers/social services providing the backbone of the care in terms of everyday interactions and time spent with patients. Although our data are based on some cost assumptions, it adds weight to the literature that EoL at-home care may be costeffective for families, especially when compared to hospital care, and provides evidence that those in the last days of life require additional support from health and social care. The findings from this study lead us to conclude that at-home support care should be made more available in England, and may provide a cost effective care for those at EoL. Further studies are required to develop the methods to capture this data further, with larger prospective studies needed.

\section{Funding Statement}

This work was supported by the Rayne Foundation, the Wates Foundation and The Burdett Trust for Nursing. 


\section{Declaration of Interest Statement}

The authors declare that they have no conflict of interests.

\section{References}

Bentur, N., Resnizky, M.S., Balicer, R., \& Eilat-Tsanani, T. (2014). Utilization and cost of services in the last 6 months of life in patients with cancer- with and without home hospice care. American Journal of Hospice and Palliative Medicine, 31, 723-25.

Bekelman, J.E., Halpern, S.D., Blankart, C.R., Bynum, J,P., Cohen, J., Fowler, R.,... Emanuel, E.J. (2016). Comparison of the site of death, health care utilization, and hospital expenditures for patients dying with cancer in 7 developed countries. JAMA, 315, 272-283. doi:https://doi.org/10.1001/jama.2015.18603.

Butler, C., Brigden, C., Gage, H., Willians, P., Holdsworth, L., Greene, K., Wee, B., Barclay, S., \& Wilson, P. (2018). Optimum hospice-at-home services for end-of-life care: protocol of a mixed-methods study employing realist evaluation. BMJ Open, 8:e021192.doi:10.1136/bmjopen-2017-021192.

Curtis, L., \& Burns, A. (2015). Unit Costs of Health and Social Care 2015. University of Kent, Canterbury: Personal Social Services Research Unit 2015.

Curtis, L., \& Burns, A. (2016). Unit Costs of Health and Social Care 2016. University of Kent, Canterbury: Personal Social Services Research Unit.

Data.Gov.UK. (2015). NHS Hospital Stay. Retrieved from Data.Gov.UK website: https://data.gov.uk/data-request/nhs-hospital-stay

Department of Health. (2008). End of life strategy: Promoting high quality care for all adults at the end of life. London: Department of Health.

Gardiner, C., Ryan, T., Gott, M. (2018). What is the cost of palliative care in the UK? A systematic review. BMJ Supportive \& Palliative Care, 8, 250-257. 
Georghiou, T., \& Bardsley, M. (2014). Exploring the cost of care at the end of life; Research report. London: Nuffield Trust.

Georghiou, T., Davies,S., Davies, A., \& Bardsley,M. (2012). Understanding patterns of health and social care full report final. London: Nuffield Trust.

Gomes, B., Calanzani, N., Gysels, M., Hall, S., \& Higginson, I.J. (2013). Heterogeneity and preferences for dying at home: A systematic review. BMC Palliative Care, 12, 7 20.

Gomes, B., Calanzani, N., Curiale, V., McCrone, P., \& Higginson, I.J. (2013). Effectiveness and cost-effectiveness of home palliative care services for adults with advanced illness and their caregivers. Cochrane Database Systematic Reviews, 6, CD 007760.

Gomes. B., \& Higginson, I.J. (2006). Factors influencing death at home in terminally ill patients with cancer: Systematic review. British Medical Journal, 332, 515-521. Hoare, S., Morris, Z.S., Kelly, M.P., Kuhn, I., \& Barclay, S. (2015). Do patients want to die at home? A systematic review of the UK literature, focused on missing preferences for place of death. PLoS ONE, 10(11), e0142723. https://doi.org/10.1371/journal.pone.0142723 Langton, J.M., Blanch, B., Drew, A.K., Hass, M., Ingham, J.M., \& Pearson, S.A. (2014). Retrospective studies of end-of-life resource utilization and costs in cancer care using health administrative data: A systematic review. Palliative Medicine, 28, 11671196.

Lustbader, D., Mudra, M., Romano, C., Lukoski, E., Chang, A., Mittelberger, J., ... Cooper, D. (2017). The impact of a home-based palliative care program in an accountable care organization. Journal of Palliative Medicine, 20(1), 23-28. doi:10.1089/jpm.2016.0265. 
McCaffrey, N., Agar, M., Harlum, J., Karnon, J., Currow, D., \& Eckermann, S.

(2013). Is home-based palliative care cost-effective? An economic evaluation of the Palliative Care Extended Packages at Home (PEACH) pilot. BMJ Supportive \& Palliative Care, 3, 431-435.

Macmillan Cancer Support. (2016). The cost of Macmillan's services fact sheet. London: Macmillan.

Marie Curie Cancer Care. (2013). We know about end of life care. Understanding the cost of end of life care in different settings. REtreived from Marie Curie Cancer Care website:

https://www.mariecurie.org.uk/globalassets/media/documents/commissioning-our$\underline{\text { services/publications/understanding-cost-end-life-care-different-settingspdf }}$

Marie Curie Cancer Care. (2014). The crisis facing terminally ill people and their families. Briefing. London: Marie Curie Cancer Care.

Marie Curie Cancer Care. (2015). The hidden costs of caring. London: Marie Curie Cancer Care.

May, P., \& Cassel, B. (2018). Economic outcomes in palliative and end-of-life care: current state of affairs. Annals of Palliative Medicine, 7 (suppl 3), s244-248

PayScale. (2017a). Care Worker Salary. Retreived from PayScale website: http://www.payscale.com/research/UK/Job=Care Worker/Hourly Rate

PayScale. (2017b). Podiatrist Salary. Retreived from PayScale website: http://www.payscale.com/research/UK/Job=Podiatrist/Salary

Public Health England. (2017). Cost-effective commissioning of end of life care. Understanding the health economics of palliative and end of life care. London: Public Health England. 
Public Health England. (2018). Statistical commentary: End of life care profiles, Febraury 2018 update. Retrieved from Public Health England website: https://www.gov.uk/government/publications/end-of-life-care-profiles-february-2018update/statistical-commentary-end-of-life-care-profiles-february-2018-update

Robinson, J., Boyd, M., O'Callaghan, A., Laking, G., Frey, R., Raphaell, D., Snow, B., \& Gott, M. (2015). The extent and cost of potentially avoidable admissions in hospital inpatients with palliative care needs: a cross-sectional study. BMJ Supportive \& Palliative Care, 5, 266-272.

Rowland, C., Hanratty, B., Pilling, M., van den Berg, B., \& Grande, G. (2017). The contributions of family care-givers at end of life: A national post-bereavement census survey of cancer carers' hours of care and expenditures. Palliative Medicine, 31, 346355.

Sixsmith, J., Ward, A., \& Youell, J. (2017). Exploring the value of providing responsive and home-based end-of-life care: A study of the Rennie Grove Night Service. Buckinghamshire: Rennie Grove Hospice Care.

UK Care Guide. (2016). Home care costs - We will help you work out what care will cost you. Retreived from UK Care Guide website: https://ukcareguide.co.uk/home$\underline{\text { care-costs/ }}$

Woodman, C., Baillie, J., \&Sivell, S. (2016). The preferences and perspectives of family caregivers towards place of care for their relatives at the end of life. A systematic review and thematic synthesis of the qualitative evidence. BMJ Supportive and Palliative Care, 6, 418-429. 
Table 1: Summary of visits and $\operatorname{costs}^{\wedge}$ for each type of healthcare worker

\begin{tabular}{|c|c|c|c|c|c|c|c|c|c|}
\hline & Visits (n) & Visits (\%) & hrs & mins & $£$ & $\$$ & $\begin{array}{l}\% \text { of } \\
\text { Cost }\end{array}$ & Cost $\mathrm{p} / \mathrm{hr}(\mathfrak{f})^{\wedge}$ & $\operatorname{Cost} \mathrm{p} / \mathrm{hr}\left(\mathbb{\$}^{\#}\right)^{\wedge}$ \\
\hline HatH service & 175 & $24.70 \%$ & 165 & 44 & $£ 7,364.14$ & $\$ 9,098.17$ & $36.50 \%$ & $£ 44.30$ & $\$ 54.73$ \\
\hline General practice & 35 & $4.90 \%$ & 14 & 37 & $£ 3,176.25$ & $\$ 3,924.16$ & $15.70 \%$ & $£ 225$ & $\$ 277.98$ \\
\hline McMillan/Marie Curie & 12 & $1.70 \%$ & 99 & 10 & $£ 2,776.67$ & $\$ 3,430.49$ & $13.80 \%$ & $£ 28$ & $\$ 34.59$ \\
\hline Private carers & 6 & $0.80 \%$ & 12 & 30 & $£ 219.38$ & $\$ 271.04$ & $1.10 \%$ & $£ 17.55$ & $\$ 21.68$ \\
\hline $\begin{array}{l}\text { Ambulance } \\
\text { (see, treat and refer) }\end{array}$ & 1 & $0.30 \%$ & & 45 & $£ 180.00$ & $\$ 222.38$ & $0.90 \%$ & $\begin{array}{r}£ 180 \\
\text { (per visit) }\end{array}$ & $\$ 222.38$ \\
\hline Rapid Response & 7 & $1.00 \%$ & 3 & 30 & $£ 276.50$ & $\$ 341.61$ & $1.40 \%$ & $£ 79$ & $\$ 97.60$ \\
\hline Carer assessment & 1 & $0.10 \%$ & 0 & 45 & $£ 27.00$ & $\$ 33.36$ & $0.10 \%$ & $£ 36$ & $\$ 44.48$ \\
\hline
\end{tabular}

* Some visits were recorded as having two formal carers, costs have been included for two carers where this was indicated.

${ }^{\wedge}$ Cost tarrifs correct at time of data analysis and do not include travel time

\# US dollar conversion rate 1.235 as of $24^{\text {th }}$ April 2020 
Table 2: Cost of visit-based care and no days of care provision per patient*

\begin{tabular}{|c|c|c|c|c|c|}
\hline Patient & $\begin{array}{l}\text { Cost of } \\
\text { care }(£)\end{array}$ & $\begin{array}{l}\text { Cost of } \\
\text { care }(\$)\end{array}$ & $\begin{array}{l}\text { No } \\
\text { Days }\end{array}$ & $\begin{array}{l}\text { Ave per } \\
\text { day }(£)\end{array}$ & $\begin{array}{l}\text { Ave per } \\
\text { day }(\$)\end{array}$ \\
\hline 1 & $£ 89.48$ & $\$ 110.55$ & 3 & $£ 29.83$ & $\$ 36.85$ \\
\hline 2 & $£ 1,220.13$ & $\$ 1,507.43$ & 18 & $£ 67.79$ & $\$ 83.75$ \\
\hline 3 & $£ 264.10$ & $\$ 326.29$ & 4 & $£ 66.02$ & $\$ 81.57$ \\
\hline 4 & $£ 105.68$ & $\$ 130.56$ & 4 & $£ 26.42$ & $\$ 32.64$ \\
\hline 5 & $£ 2,276.33$ & $\$ 2,812.34$ & 20 & $£ 113.82$ & $\$ 140.62$ \\
\hline 6 & $£ 297.08$ & $\$ 367.03$ & 3 & $£ 99.03$ & $\$ 122.35$ \\
\hline 7 & $£ 998.23$ & $\$ 1,233.28$ & 11 & $£ 90.75$ & $\$ 112.12$ \\
\hline 8 & $£ 485.70$ & $\$ 600.07$ & 18 & $£ 26.98$ & $\$ 33.33$ \\
\hline 9 & $£ 794.26$ & $\$ 981.28$ & 13 & $£ 61.10$ & $\$ 75.49$ \\
\hline 10 & $£ 1,034.49$ & $\$ 1,278.08$ & 14 & $£ 73.89$ & $\$ 91.29$ \\
\hline 11 & $£ 1,169.61$ & $\$ 1,445.02$ & 12 & $£ 97.47$ & $\$ 120.42$ \\
\hline 12 & $£ 33.23$ & $\$ 41.05$ & 1 & $£ 33.23$ & $\$ 41.05$ \\
\hline 13 & $£ 1,059.39$ & $\$ 1,308.84$ & 12 & $£ 88.28$ & $\$ 109.07$ \\
\hline 14 & $£ 132.90$ & $\$ 164.19$ & 2 & $£ 66.45$ & $\$ 82.10$ \\
\hline 15 & $£ 638.61$ & $\$ 788.98$ & 14 & $£ 45.62$ & $\$ 56.36$ \\
\hline 16 & $£ 84.70$ & $\$ 104.64$ & 16 & $£ 5.29$ & $\$ 6.54$ \\
\hline 17 & $£ 1,132.17$ & $\$ 1,398.76$ & 12 & $£ 94.35$ & $\$ 116.57$ \\
\hline 18 & $£ 279.94$ & $\$ 345.86$ & 14 & $£ 20.00$ & $\$ 24.71$ \\
\hline 19 & $£ 1,071.58$ & $\$ 1,323.90$ & 14 & $£ 76.54$ & $\$ 94.56$ \\
\hline 20 & $£ 319.60$ & $\$ 394.86$ & 14 & $£ 22.83$ & $\$ 28.21$ \\
\hline 21 & $£ 697.31$ & $\$ 861.51$ & 14 & $£ 49.81$ & $\$ 61.54$ \\
\hline 22 & $£ 692.05$ & $\$ 855.01$ & 7 & $£ 98.86$ & $\$ 122.14$ \\
\hline 23 & $£ 354.92$ & $\$ 438.49$ & 7 & $£ 50.70$ & $\$ 62.64$ \\
\hline 24 & $£ 168.74$ & $\$ 208.47$ & 14 & $£ 12.05$ & $\$ 14.89$ \\
\hline 25 & $£ 540.61$ & $\$ 667.91$ & 14 & $£ 38.62$ & $\$ 47.71$ \\
\hline 26 & $£ 1,409.84$ & $\$ 1,741.82$ & 13 & $£ 108.45$ & $\$ 133.99$ \\
\hline 27 & $£ 525.70$ & $\$ 649.49$ & 6 & $£ 87.62$ & $\$ 108.25$ \\
\hline 28 & $£ 822.35$ & $\$ 1,015.99$ & 14 & $£ 58.74$ & $\$ 72.57$ \\
\hline 29 & $£ 90.20$ & $\$ 111.44$ & 14 & $£ 6.44$ & $\$ 7.96$ \\
\hline 30 & $£ 1,403.36$ & $\$ 1,733.81$ & 11 & $£ 127.58$ & $\$ 157.62$ \\
\hline Overall & $£ 20,192.26$ & $\$ 24,946.93$ & 333 & $£ 60.64$ & $\$ 74.92$ \\
\hline
\end{tabular}

*Costs are for staff time only and do not include travel costs, equipment, medication or other supplies used during the visit. 
Table 3: Number of visits by General Practitioners, District Nurses and HatH, by deaths and non-deaths during study period

\begin{tabular}{|l|l|l|l|l|l|l|}
\hline & $\begin{array}{l}\text { Individual } \\
\text { died during } \\
\text { study n=14 }\end{array}$ & $\begin{array}{l}\text { Average } \\
\text { visit per } \\
\text { person }\end{array}$ & $\begin{array}{l}\text { Individual } \\
\text { did not die } \\
\text { during } \\
\text { study } \\
\mathrm{n}=16\end{array}$ & $\begin{array}{l}\text { Average } \\
\text { visit per } \\
\text { person }\end{array}$ & $\begin{array}{l}\text { Total } \\
\text { Visits } \\
\mathrm{n}=30\end{array}$ & $\begin{array}{l}\text { Average } \\
\text { visit per } \\
\text { person }\end{array}$ \\
\hline $\begin{array}{l}\text { General } \\
\text { Practitioner visits }\end{array}$ & 21 & 1.5 & 14 & 0.9 & 35 & 1.2 \\
\hline $\begin{array}{l}\text { District nurse } \\
\text { visits }\end{array}$ & 60 & 4.3 & 57 & 3.6 & 117 & 3.9 \\
\hline HatH visits & 104 & 7.4 & 71 & 4.4 & 175 & 5.8 \\
\hline
\end{tabular}

Figure 1: Night service template 
Thtal Mileage:

Total number of deaths attended - Herts:

Total number of incoming calls:

Date of call/visit:

Identify Herts or Bucks patients and put " if patient surgery is one of the following: (surgery names removed)

Patient ID

Underline if individual is resides in a care/nursing home

Who has called? (Carer/Patient/HCA)

Time of call

Reason for Incoming Call

1. Planned visit from late shift

2. Symptom control

a. Pain

b. Anxiety

c. Nausea/vomiting

d. Other please specify

3. Carer support

4. Death

5. Death verified by night team Yes/No

6. Message

7. Other please specify

8. Advice required by other HCP

\section{Reason for Not Visiting}

1. Patient/carer reassured/settled by tel advice

2. Managing another patient/ unable

3. Other service required

4. Incoming message/feedback

5. End of shift/time management

Passed to other HCP/Service

1. Early shift nurse

2. DN service

3. GP service

4. Ambulance service

5. Hospital team

6. Palliative care service

7. Other please specify

\section{Reason call passed on to other HCP}

1. Prescription needs

2. New symptom/unexpected change

3. Urgent medical attention necessary

4. Involved with another patient

5. Admission necessary

\section{Reason for Hospital Admission}

1. Complex acute symptoms

2. Discharge complications

3. Patient/carer choice

4. Carer breakdown

5. Other

Was hospital admission prevented Yes/No

Rationale - see key \& record number

Total Travel Time:

Bucks:

Bucks*:

Total number of hospital admissions:

Staff name \& band

\begin{tabular}{|l|l|l|l|l|l|l|}
\hline H/B & H/B & H/B & H/B & H/B & H/B & H/B \\
\hline & & & & & & \\
\hline & & & & & & \\
\hline & & & & & &
\end{tabular}

\begin{tabular}{l|l|l|l}
\hline & & & \\
\hline
\end{tabular}

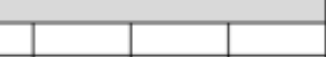

Figure 2: Cost of care per day by those who died during the data collection period 


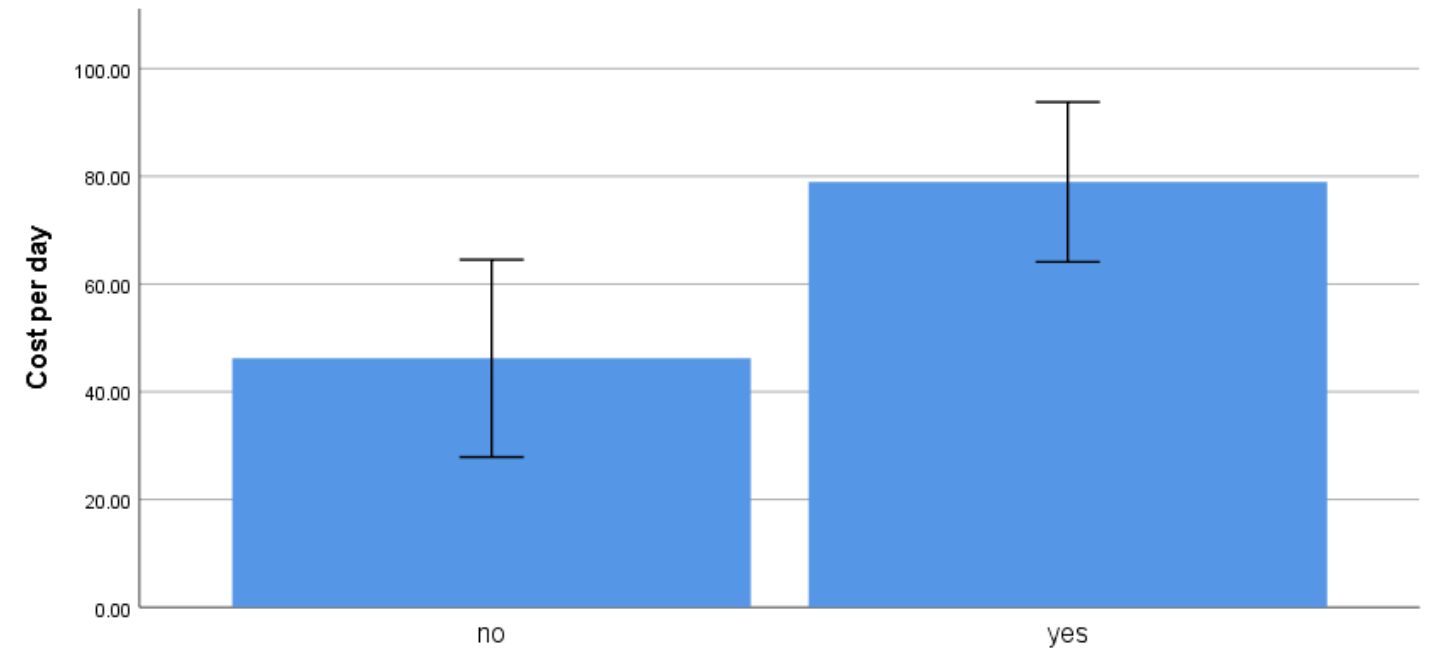

Died during data collection

Error Bars: $95 \% \mathrm{Cl}$ 